\title{
удК 517.977 .58
}

\section{P. TEHHO}

\section{ПРИБЛИЖЕННО ОПТИМАЛЬНОЕ УПРАВЛЕНИЕ БИЛИНЕЙНОЙ СИСТЕМОЙ}

R. TENNO. BILINEAARSE SUSTEEMI LAHISOPTIMAALNE JUHTIMINE

R. TENNO. SUB-OPTIMAL CONTROL OF A BILINEAR SYSTEM

$$
\text { (Представил Н. Алумяэ) }
$$

Билинейные системы являются наиболее простым обобщением линейных. Несмотря на это, возникают серьезные трудности в связи с построением оптимальных управлений. В некоторых случаях эти трудности могут быть преодолены - найдены управления, которые аппроксимируют оптимальные лучше, чем предложенные в $\left[{ }^{1}\right]$.

Ставится задача. Найти стратегию $\left(\alpha_{t}\right)$ управления с обратной связью $\alpha_{t}=\alpha_{t}\left(\xi_{0}, \ldots, \xi_{t}\right)$ такую, которая минимизирует функционал

$$
v^{\alpha}=M\left\{\sum_{t=0}^{N-1}\left(\Theta_{t}^{2}+\alpha_{t}^{\mathrm{r}} \Upsilon \alpha_{t}\right)+\Theta_{N}^{2}\right\},
$$

если управляемая $\left(\Theta_{t}\right)$ и наблюдаемая последовательности $\left(\xi_{t}\right)$ заданы уравнениями

$$
\begin{aligned}
& \Theta_{t+1}=\mu+\alpha_{t}^{\mathrm{T}} G \Theta_{t}+\sigma \varepsilon_{t+1}, \quad \Theta_{0}=\tilde{\Theta}, \\
& \xi_{t+1}=F\left(t, \xi_{t}\right)+A \Theta_{t}+e_{t+1}, \quad \xi_{0}=\xi_{0},
\end{aligned}
$$

где $\varepsilon_{t}, e_{t}$ - независимые гауссовские величины, $\xi_{0}-$ фиксированное, $\tilde{\Theta}-$ случайное начальное условие с гауссовской плотностью распределения со средним $m_{0}$ и дисперсией $\gamma_{0}, F\left(t, \xi_{t}\right)$ - функция, имеющая конечный момент второго порядка.

Решив двухшаговую $(N=2)$ задачу, получим следующий результат.

Пусть матрица $\Upsilon$ положительно определена. Тогда задача (1), (2) имеет решение. Оптимальное управление на первом шаге определяется из решения уравнения

$$
S_{\alpha}+R_{\alpha}=0
$$

при условии, что матрица $S_{\alpha \alpha}+R_{\alpha \alpha}$ положительно определена. Здесь $S_{\alpha}, R_{\alpha}$ - первые, $S_{\alpha \alpha}, R_{\alpha \alpha}-$ вторые производные от функции

$$
S(\alpha)=m^{2}+\gamma+\alpha^{\mathrm{T}} \Upsilon \alpha
$$

и

$$
R(\alpha)=c^{2}+C+\sigma^{2}+\sqrt{\pi} \mu^{2} Y \operatorname{Re} w(z),
$$

$m=M\left(\Theta_{0} / \xi_{0}\right)$ - условное среднее, $\gamma=\operatorname{cov}\left(\Theta_{0} / \xi_{0}\right)=$ условная ковариация, 


$$
c=\mu+\alpha^{\mathrm{T}} G m
$$

- прогноз состояния $\Theta_{1}$,

- дисперсия прогноза

$$
C=\sigma^{2}+\left(\alpha^{\mathrm{T}} G\right)^{2} \gamma
$$

$$
w(z)=e^{-z^{2}}[1-\operatorname{erf}(-i z)]
$$

- интеграл вероятности от комплексного аргумента $z=X+i Y$. Переменная $z$ связана с остальными параметрами задачи (1), (2) по формулам

$$
\begin{gathered}
\left.\sqrt{2} X T=-c, \quad 2 Y^{2} T^{2} G^{\mathrm{T}}\left[\Upsilon+G G^{\mathrm{T}}\left(C-T^{2}\right)\right]^{-1}\right) G=1 ; \\
T \sqrt{1+A^{\mathrm{T}} \gamma A}=\gamma A G^{\mathrm{T}} \alpha .
\end{gathered}
$$

Оптимальное управление на втором шаге единственное. Оно задается равенством

$$
\alpha^{*}=-\left[\Upsilon+G G^{\mathrm{r}}\left(m_{1}^{2}+\gamma_{1}\right)\right]^{-1} G m_{1} \gamma,
$$

где $m_{1}=M\left(\Theta_{1} / \xi_{1}\right), \gamma_{1}=\operatorname{cov}\left(\Theta_{1} / \xi_{1}\right)$.

В общем случае, когда $N$ любое натуральное число, решение задачи (1), (2) не мыслимо ввиду сложности. Приближенно она может быть решена следующим образом. Пусть $m_{t}-$ условное среднее, $\gamma_{t}-$ условная ковариация, вычисляемые согласно уравнениям фильтрации $\left[{ }^{2}\right]$. Примем $m=m_{t}, \gamma=\gamma_{t}$ в (3) и $m_{1}=m_{N-1}, \gamma_{1}=\gamma_{N-1}$ в (4). Тогда оптимальное в смысле

$$
v_{1}^{\alpha}=M\left\{\sum_{\tau=t}^{t+1}\left(\Theta_{\tau}^{2}+\alpha_{\tau}^{\mathrm{T}} \Upsilon \alpha_{\tau}\right)+\Theta_{\tau+2}^{2}\right\}
$$

управление на каждом шаге $t=0,1, \ldots, N-2$ определяется из уравнения (3), а на $t=N-1$ из равенства (4).

\section{Л ИТЕРА Т Р А}

1. Jacobs, O. L. R., Potter, R. V. In: Recent Theoretical Developments in Control (Ed. by M. J. Gregson). London-New York-San Francisco, Academic Press, $1978,403-419$.

2. Липцер Р. Ш., Ширяев А. Н. Статистика случайных процессов. М., «Наука», 1974.

Институт кибернетики

Академии наук Эстонской ССР
Поступила в редакцию 11/II 1986 